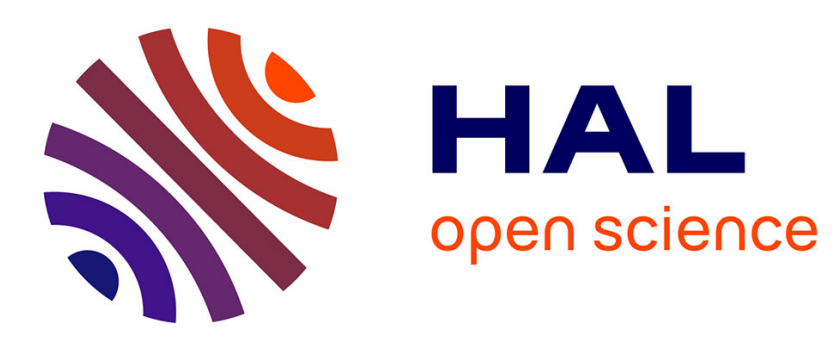

\title{
Micronic thermal characterization of vertical interfaces using the photoreflectance method
}

F. Lepoutre, Ph. Forge, J. Joulaud, L. Paradis, D. Rochais

\section{To cite this version:}

F. Lepoutre, Ph. Forge, J. Joulaud, L. Paradis, D. Rochais. Micronic thermal characterization of vertical interfaces using the photoreflectance method. Journal de Physique IV Proceedings, 1994, 04 (C7), pp.C7-571-C7-574. 10.1051/jp4:19947134 . jpa-00253189

\section{HAL Id: jpa-00253189 https://hal.science/jpa-00253189}

Submitted on 1 Jan 1994

HAL is a multi-disciplinary open access archive for the deposit and dissemination of scientific research documents, whether they are published or not. The documents may come from teaching and research institutions in France or abroad, or from public or private research centers.
L'archive ouverte pluridisciplinaire HAL, est destinée au dépôt et à la diffusion de documents scientifiques de niveau recherche, publiés ou non, émanant des établissements d'enseignement et de recherche français ou étrangers, des laboratoires publics ou privés. 


\title{
Micronic thermal characterization of vertical interfaces using the photoreflectance method
}

\author{
F. Lepoutre, Ph. Forge, J.L. Joulaud, L. Paradis* and D. Rochais* \\ ONERA, L3C, BP. 79, 92322 Châtillon, France \\ * CEA, BP. 12, 91680 Bruyères-le-Châtel, France
}

\begin{abstract}
This paper presents the theoretical and experimental possibilities of a modulated photothermal method, laser induced photoreflectance, for inspecting the quality of interfaces with micronic spatial resolutions. An analytical model is established for semi-infinite materials containing an internal interface perpendicular to the sample surface. The presented application is the detection of thermal resistances in damaged materials.
\end{abstract}

\section{INTRODUCTION}

The method described in this paper is called laser-induced photoreflectance and is related to the photothermal methods (see figure 1). Its principle is to detect the periodic temperature through the variations in thermally induced modulations of the optical reflection coefficient of the surface.

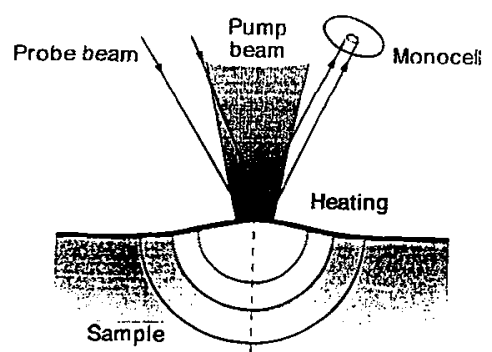

Figure 1

Principle of the photoreflectance method. The pump beam is modulated. The probe is continuous. The probe beam must not be stopped down after its reflection by the sample surface in order not to detect the thermoelastic deformation.

The goal of this paper is to demonstrate that the proposed method is well suited for local investigations of interface quality. The paper is divided in two parts, one on theory (Section 2) and the other on the experiments (Section 3). To simplify the theoretical approach, we limited our study to the problem of sinusoidal heat diffusion in a semi-infinite medium containing only one interface perpendicular to the surface. This fundamental problem can be solved analytically so that a physical understanding of the respective effects of sample properties and experimental conditions is rather easy to develop. 
The results of this calculation are applied in Section 3 for the characterization of cracks.

\section{MODEL}

\subsection{Equations of the Problem}

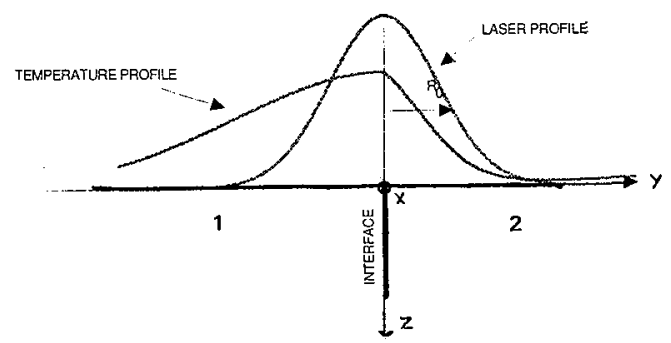

Figure 2

Geometry of the problem. The surface is the $x y$ plane and the interface is the $x z$ plane which separates media 1 and 2.

All quantities related to the media of the $y<0$ or the $y>0$ regions are subscripted 1 or 2 respectively, and 1,2 when the relation involved is valid in both regions 1 and 2 . The pump beam is located at point $(0$, $\left.y_{0}, 0\right)$ with a gaussian shape of radius $r_{p}$. The probe beam is located at point $\left(0, y_{0}, 0\right)$ with a gaussian shape of radius $r_{S}$.

The problem is to solve the heat diffusion equation with source distributions $P_{1,2}$ :

$$
\lambda_{1,2} \Delta \mathrm{T}_{1,2}-\mathrm{j} \omega \rho_{1,2} \mathrm{C}_{1,2} \mathrm{~T}_{1,2}+\mathrm{P}_{1,2}=0
$$

in the media 1 and 2 with the boundary conditions:

at $\mathrm{z}=0$

$$
-\lambda_{1,2} \frac{\partial \mathrm{T}_{1,2}}{\partial \mathrm{z}}\left(\mathrm{z}=0^{-}\right)=0
$$

and at $\mathrm{y}=0$

$$
-\lambda_{1} \frac{\partial \mathrm{T}_{1}}{\partial \mathrm{y}}\left(\mathrm{y}=0^{-}\right)=-\lambda_{2} \frac{\partial \mathrm{T}_{2}}{\partial \mathrm{y}}\left(\mathrm{y}=0^{+}\right)
$$

$$
\mathrm{T}_{1}\left(\mathrm{y}=0^{-}\right)-\mathrm{T}_{2}\left(\mathrm{y}=0^{+}\right)=-\mathrm{R}_{\mathrm{T}} \lambda_{1} \frac{\partial \mathrm{T}_{1}}{\partial \mathrm{y}}\left(\mathrm{y}=0^{-}\right)
$$

In practice, the case of semi transparent materials is difficult to study since the local optical absorption cannot be known precisely in a complex material such as a composite. As a result, the problem will be simplified in the next section by taking the absorption as infinite.

\subsection{Solution}

To find the general solution of equation (1), it is useful to examine the symmetry of the problem. With a delta function as source term, the temperature distribution has a cylindrical symmetry around the axis which passes through the point source and is perpendicular to the plane of the crack. Since the crack plane is the $\mathrm{xz}$ plane, let us call $\mathrm{y}$ this axis. The usual mathematical tool corresponding to this cylindrical symmetry is the Hankel transform vs the distance to this $y$ axis, i.e. $\rho=\sqrt{ }\left(\left(x^{2}-x^{\prime 2}\right)+\left(z^{2}-z^{\prime}\right)\right)$

$$
\widetilde{T}(\beta, y)=\int_{0}^{\infty} J_{0}(\beta \rho) T(y, \rho) \rho d \rho
$$

The general solution can be written as the sum of the homogeneous solution (equation (1) without source term) and a particular solution of equation (1):

$$
\widetilde{T_{1,2}}(\beta, y)=A_{1,2} e^{-m_{1,2} y}+B_{1,2} e^{m_{1,2} y}+\frac{1}{2 \pi \lambda_{1,2}} \frac{e^{-m_{1,2}\left|y-y^{\prime}\right|}}{m_{1,2}}
$$


with the wave numbers $\mathrm{m}_{1,2}$ given by :

$$
m_{1,2}=\sqrt{\beta^{2}+\frac{j \omega}{\alpha_{1,2}}}
$$

The constants $A_{1}, A_{2}, B_{1}$ and $B_{2}$ are calculated using the Hankel transform of the boundary conditions ( 2 and 3$)$.

The following steps consist in a return to the normal space, by taking the inverse Hankel transform

$\mathrm{T}_{\mathrm{i}}^{\mathrm{j}}$ of the four expressions $\widetilde{\mathrm{T}_{\mathrm{i}}^{\mathrm{j}}}$ (j meaning that the temperature must be calculated with the delta function source either in medium 1 or in medium 2) followed by their convolutions with the gaussian shapes of the actual source and probe.

\subsection{Results}

The phase temperature distribution deduced from this model is graphed in figure 3 for a vertical interface in a homogeneous sample.

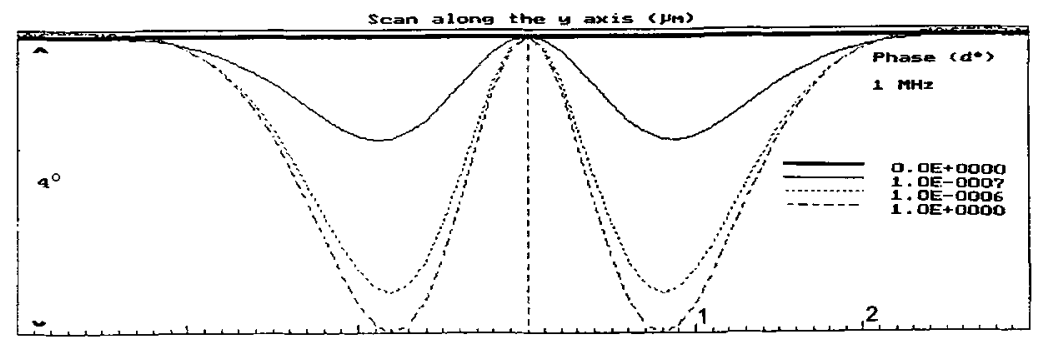

Figure 3

Phase variations of the periodic temperature calculated at $1 \mathrm{MHz}$ when the two merged beams $\left(\mathrm{r}_{\mathrm{s}}=\mathrm{r}_{\mathrm{p}}=\right.$ $1 \mu \mathrm{m}$ ) scan the surface of a medium of diffusivity $410^{-6} \mathrm{~m}^{2} \mathrm{~s}^{-1}$, and conductivity $10 \mathrm{Wm}^{-1} \mathrm{~K}^{-1}$ containing a thermal resistance in the $(\mathrm{x}, \mathrm{z})$ plane varying from 0 to $\infty$ (see insert for the resistance values ).

The thermal resistance produces one amplitude maximum and one phase variation minimum on each side of the crack. This result is quite similar to those obtained by other authors, Mc. Donald [1], Rantala [2]. The sensitivity of the phase contrast to changes of $f$ or of $r_{0}$ is not very large and, when the beams are merged, the contrast in phase is never larger than a few degrees, even for an infinite resistance. The best contrast in phase is obtained when the frequency is close to a value $f_{0}$ defined by :

$$
\mathrm{f}_{0}=\frac{\alpha}{\pi \mathrm{r}_{0}^{2}}
$$

which, physically, corresponds to the equality between the thermal diffusion length $\mu$ in the medium and an effective pump beam radius $r_{0}$ given by:

$$
\mathrm{r}_{0}^{2}=\mathrm{r}_{\mathrm{s}}^{2}+\mathrm{r}_{\mathrm{p}}^{2}
$$

\section{EXPERIMENTS}

\subsection{Experimental Setup}

The experimental setup described in this section uses the principle which was explained in figure 1. The main difference between this principle and the practical setup is that the probe and pump axis are actually colinear.

The pump beam comes from an argon laser modulated by an acousto-optic modulator. The power of the pump can be varied from 10 milliwatts to a few watts. The modulation frequency is adjustable from 0 to $50 \mathrm{MHz}$. The probe used is a nonpolarised He Ne laser of a power of a few milliwatts chosen for its good pointing stability. The signal detected by the photodiode is sent to a lock-in amplifier (working at frequencies up to $50 \mathrm{MHz}$ ) which also receives a reference signal from the modulator. The 
signal finally delivered by the lock-in is the amplitude and/or the phase of the fundamental Fourier component of the modulated part of the temperature increase produced by the pump heating.

Both beams are focused by a metallurgical microscope at the limit of its optical resolution ( 0.5 $\mu \mathrm{m})$. They are mixed using a dichroic plate and, after reflection on the sample, the probe beam is sent to the photocell by a beam splitter. A Faraday cell prevents any return of the reflected probe beam into the cavity. The pass band of the photodiode is about $20 \mathrm{MHz}$.

\subsection{Cracks}

The resistive interface between two parts of a same medium is well represented, in practice, by a crack.

We prepared a sample of black glass which was microindented with a diamond tool. This was done because, in such materials, microindentation produces very tight cracks perpendicular to the surface. The air gaps between the edges of the cracks are generally less than $1 \mu \mathrm{m}$.

Figure $4 \mathrm{a}$ represents the photothermal phase recorded in a small area close to the crack.

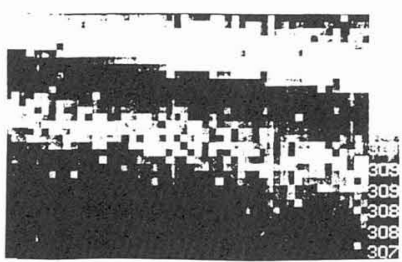

a

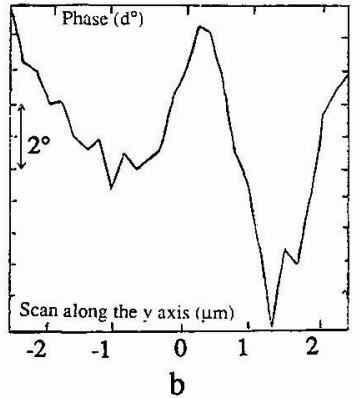

Figure 4

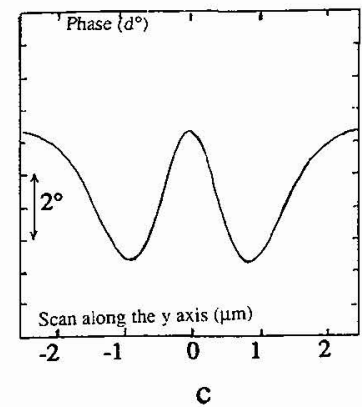

c

Crack in a black ceramic. $1 \mathrm{MHz} . \mathrm{r}_{0}=1 \mu \mathrm{m} . \alpha=10^{-6} \mathrm{~m}^{2} \mathrm{~s}^{-1}$.

a/ phase image $(5 \times 3 \mu \mathrm{m})$. The crack is located between the two white lines.

$\mathrm{b} /$ phase variations along a line crossing the crack located at $\mathrm{y}=0$.

$\mathrm{c} /$ calculation corresponding to the data of the experiment.

The phase exhibits the two predicted extrema from part to part of the crack (see figure $4 \mathrm{~b}$ ). In figure $4 \mathrm{c}$, the phase shift, calculated with the parameters of the experiment, the sample thermal properties, and $R_{T} \infty$, is roughly equal to half the experimental value. The explanation for this disagreement is most likely to be found in the semi-transparency of the sample, which can play a non-negligible role at the used frequency.

\section{CONCLUSION}

We have presented an experimental setup designed to characterize the thermal behaviour of vertical interfaces. In principle, these measurements can be quantitative provided the thermal and optical properties of the tested sample are known.

We performed a calculation of the periodic temperatures induced in a media containing an interface perpendicular to its surface. Such a calculation, though academic, is important because it is actually the first step towards understanding of the microscopic thermal behaviour of interfaces such as the ones encountered in multilayers, damaged materials, composites, and so on.

\section{REFERENCES}

[1].F. A. Mc Donald, G. C. Wetsel and G. E. Jamieson, "Photothermal beam deflection of vertical interfaces", Can. J. Phys. 64, 1265-1268 (1986).

[2].J. Rantala, J. Hartikainen and J. Jaarinen, "Finite elements calculation compared with photothermal craks measurements", Appl. Phys. A 50, 465-471 (1990). 